TEMAS EMERGENTES

\title{
El derecho humano a la ciencia: Un viejo derecho con un gran futuro
}

\author{
The human right to science: An old right with a great future
}

\begin{abstract}
Mikel MANCISIDOR
Comité de Derechos Económicos, Sociales y Culturales, Naciones Unidas, Suiza
\end{abstract}

\begin{abstract}
RESUMEN Cuando hablamos del derecho humano a la ciencia se puede pensar que nos referimos a un derecho nuevo, creado recientemente para hacer frente a los desafíos que la ciencia y la tecnología generan en nuestra sociedad del siglo XXI. Sin embargo, el derecho a la ciencia es un derecho ya recogido en la Declaración Universal de los Derechos Humanos de 1948 y en el Pacto Internacional de Derechos Económicos, Sociales y Culturales de 1966. Lo podemos encontrar incluso antes, en el sistema regional interamericano, en particular, en la Carta de la Organización de los Estados Americanos y en la Declaración Americana de Derechos y Deberes del Hombre. Pocos saben que, de hecho, los países lationamericanos, en general, y Chile, muy en particular, tuvieron un papel crucial en el reconocimiento universal de este derecho. La primera parte del artículo está dedica a explicar la historia de este derecho y su reconocimiento jurídico internacional. En una segunda parte estudiaremos su situación institucional actual en el ámbito de las Naciones Unidas y, finalmente, en la tercera parte indagaremos en las características de este derecho, su contenido normativo, elementos y tipo de obligaciones que crea.
\end{abstract}

PALABRAS CLAVE Derecho a la ciencia, derechos culturales, derechos humanos.

ABSTRACT When we talk about the human right to science, many may think that we are speaking about a new right, recently created to face the challenges that science and technology generate in our society of the 21st century. However, the right to science is already enshrined in the Universal Declaration of Human Rights (1948) and in the International Covenant on Economic, Social and Cultural Rights (1966). We can find it even earlier in the inter-American regional system, particularly, in the Charter of the Organization of American States (1948) and in the American Declaration of the Rights and Duties of Man (1948). Few know that, in fact, the Latin American countries in general and Chile in particular played a crucial role in the universal recognition of this 
right. The first part of the article explains the history of the right to science and its international legal recognition. In a second chapter, we will study its current institutional situation within the United Nations and, finally, in the third chapter, we will analyze the characteristics of this right, its normative content, elements and type of obligations that it creates.

KEYWORDS Right to science, culture rights, human rights.

\section{Introducción}

El derecho humano a la ciencia es un gran desconocido incluso entre muchos activistas, académicos y expertos en derechos humanos. Ha sido un derecho por mucho tiempo olvidado por los organismos internacionales encargados de la defensa y promoción de los derechos humanos, incluyendo a Naciones Unidas, y lo es igualmente para buen número de Estados que están obligados a proteger y fomentar este derecho.

El derecho a la ciencia pertenece al grupo de los derechos culturales. Muchas veces se ha dicho que los derechos económicos, sociales y culturales (DESC) son, por su menor desarrollo, los hermanos pobres de la familia de los derechos humanos ${ }^{1}$ Y Y dentro de los DESC, los derechos culturales son los menos elaborados y protegidos. Podríamos dar un paso más y situar el derecho a la ciencia a la cola, a su vez, de los derechos culturales. Al menos así ha sido hasta la fecha. Pero las cosas tal vez estén cambiando.

Frente a la importante literatura que en los últimos años ha desarrollado la doctrina de los DESC, son pocos los artículos consagrados al derecho a la ciencia ${ }^{2}$ y menos aún las monografías dedicadas específicamente a este derecho, lo que ha llevado a una de las mayores especialistas en este derecho a afirmar que el actual reto de desentrañar los contenidos de este derecho y su aplicabilidad corrige seis décadas perdidas sin avances (Shaver, 2010: 152).

En la práctica judicial, mientras que en los últimos años se ha dado un importante desarrollo de la jurisprudencia comparada en materia de protección y aplicación de los DESC (derecho a la vivienda o derecho al agua, por poner sólo dos ejemplos), no ha sucedido lo mismo con el derecho a la ciencia. Seguramente podemos contar con los dedos de una mano las sentencias de altos tribunales que han fundamentado sus decisiones, siquiera de manera secundaria, complementaria o como obiter dictum, en el derecho a la ciencia.

1. En Mancisidor (2010) defendimos que, si bien esta idea de los DESC como hermanos pobres fue cierta durante mucho tiempo, el abismo que separaba los derechos civiles y políticos de los DESC se va poco a poco reduciendo. Véase, además, Mancisidor, 2015a y b.

2. Dicho sea esto con el pleno conocimiento y la más alta consideración hacia los imprescindibles escritos pioneros de Richard Pierre Claude, William Schabas, Audrey R. Chapman, Yvonne Donders, Lea Shaver, Jessica Wyndham, Samantha Besson, Farida Shaheed y Margart Vitullo. 
Estamos pues ante un derecho aparentemente precario que, sin embargo, está consagrado en lo más alto del derecho internacional de los derechos humanos. No estamos ante un derecho nuevo ni secundario. Es por eso que creemos que ha llegado el momento de tomárselo en serio, sacarlo de su ostracismo, para ponerlo al día y colocarlo en igualdad de condiciones entre el resto de derechos humanos, para desplegar todas sus potencialidades que, siendo las de la ciencia, son las infinitas de la creatividad humana.

\section{Contexto histórico, el papel de Chile y el alcance del derecho}

\section{El contexto histórico y el papel de Chile}

El derecho a la ciencia fue reconocido en la Declaración Universal de Derechos $\mathrm{Hu}$ manos de 1948, en su artículo 27, de la siguiente forma: «Toda persona tiene derecho a tomar parte libremente en la vida cultural de la comunidad, a gozar de las artes y a participar en el progreso científico y en los beneficios que de él resulten».

Debemos remitirnos a los trabajos preparatorios ${ }^{3}$ de la Declaración para descubrir por qué sus redactores eligieron estas precisas palabras y, al hacerlo, qué quisieron decir ${ }^{4}$.

Para ello debemos situarnos en su momento histórico, en los primeros años de la posguerra y en el inicio de la Guerra Fría. Pero sin olvidar que ya en enero de 1941 el Presidente Roosevelt hizo referencia, en su famoso discurso de las Cuatro Libertades, al «disfrute de los frutos del progreso científico» en un contexto que nos puede permitir considerarlo como un precedente directo del derecho a la ciencia.

Ya en la posguerra, dos nuevas circunstancias influyeron gravemente en los debates sobre la ciencia: el recuerdo de las dos bombas atómicas lanzadas sobre Hiroshima y Nagasaki en agosto de 1945, que colocó la cuestión de la ciencia, sus límites, control y la responsabilidad social de los científicos en el centro de muchos debates ${ }^{5}$, y la rivalidad entre los Estados Unidos y la Unión Soviética que afectaría de forma muy viva a la cuestión de la ciencia y su papel en la esfera internacional.

Lo que luego sería la Declaración Universal de los Derechos Humanos se fue trabajando por medio de sucesivos borradores durante año y medio. El primer borrador

3. Para esta parte histórica me he basado en dos clásicos imprescindibles: Morsink (1999) y Glendon (2001), y en una obra reciente escrita por uno de los grandes expertos en el derecho humano a la ciencia: Schabas (2013).

4. Bien sabemos que, como establece el artículo 32 de la Convención de Viena sobre el Derecho de los Tratados de 1969, «los trabajos preparatorios del tratado y a las circunstancias de su celebración» no constituyen medios principales de interpretación del texto a los efectos de su aplicación, sino sólo «medios complementarios», pero sí nos sirve su estudio para conocer en profundidad este derecho.

5. Para una historia de los científicos y la bomba atómica, véase Preston (2005). 
fue el del jurista canadiense John Peters Humphrey, en el que nos encontramos ya con un derecho a la ciencia relacionado, en el mismo artículo, con la cultura y las artes. Según René Cassin (Morsink, 1999, 218), la norma fue incluida a solicitud de algunas organizaciones culturales, entre ellas la Unesco ${ }^{6}$. El derecho viene formulado en esta primera versión como derecho (right) a «participar en los beneficios de la ciencia».

Esta formulación está inspirada, como la activa delegación chilena se esmeró en recordar, en los trabajos preparatorios de la Carta de la Organización de Estados Americanos (OEA) y de la Declaración Americana de Derechos ${ }^{7}$, que terminó siendo aprobada en Bogotá poco meses ante de la Declaración Universal y que incluía un artículo que decía: «toda persona tiene el derecho de participar en la vida cultural de la comunidad, gozar de las artes y disfrutar de los beneficios que resulten de los progresos intelectuales y especialmente de los descubrimientos científicos».

La Carta de la OEA, en su artículo 38, decía incluso con ambición más general: «los Estados miembros difundirán entre sí los beneficios de la ciencia y de la tecnología, promoviendo, de acuerdo con los tratados vigentes y leyes nacionales, el intercambio y el aprovechamiento de los conocimientos científicos y técnicos».

Chile fue protagonista de la inclusión de este derecho en la Declaración Universal de los Derechos Humanos gracias al trabajo de su primer embajador ante la ONU, el diplomático Hernán Santa Cruz: destacado miembro de la Comisión de Derechos Humanos y del Comité redactor de la Declaración y, en muchos casos, líder de la posición latinoamericana (hay que recordar que América Latina aportaba 20 miembros de los 51 Estados parte de la ONU original, así que se trataba de un grupo potentísimo, especialmente en un sistema en que cada país representaba un voto). Hernán Santa Cruz fue uno de los más firmes defensores de la inclusión de los DESC en la Declaración y, muy en particular, de la inclusión del derecho de todas las personas a participar de los beneficios del avance científico.

Durante el proceso negociador de la Declaración, la formulación del derecho a la ciencia pasó desde la inicial versión basada «en los beneficios» a la más amplia idea del derecho «a participar en el avance científico». Este transcendental cambio fue aprobado a propuesta de China en base, según el propio delegado Peng Chun Chang, a la autoridad de Francis Bacon (Schabas, 2007: 276; Morsink, 199: 219)

6. Sobre la aportación intelectual de la Unesco a la redacción de la Declaración Universal, véase el documento «Human Rights: Comments and interpretations. A symposium edited by Unesco», del 25 de julio de 1948, con aportaciones de Maritain, Gandhi, E. H. Carr, B. Croce, Teilhard de Chardin, A. Huxley y dos ponencias específicas sobe temas científicos, a saber, de Jan M. Burgers, «Rights and Duties Concerning Creative Expression, in particular in Science», y de William A. Noyes, «Science and the Rights of Man». Docuemento disponible en http://bit.ly/2uyERSp.

7. Claude (2002: 250), a su vez, busca los orígenes de esa aportación en la Inter-American Conference on the Problems on War and Peace, realizada en México en 1945.

8. Es curioso que, en su fundamentación, P. C. Chang haya recurrido a la autoridad de un clásico occi- 
Sin embargo, este cambio y la pérdida de la palabra «beneficios» no terminó de convencer y rápidamente se buscó su recuperación (Morsink, 1999; Schabas 2007). La palabra fue recuperada a propuesta de Cuba con el argumento de que «no todo el mundo está suficientemente capacitado para jugar un papel en el avance científico». Los representantes de Cuba argumentaron que lo necesario era que el texto dijera que todo el mundo tiene el derecho «a participar en los beneficios que resulten del avance científico». René Cassin y Hernán Santa Cruz apoyaron la propuesta cubana. La delegaciones de China y de Arabia Saudí respondieron que, aun en ausencia de conocimientos científicos, todos tenemos la capacidad de cierto disfrute más amplio de la ciencia que sus solos beneficios directos.

El comentario de Cuba puede sugerir la idea de una renuncia a una consideración amplia del derecho a participación (activa) en la ciencia y la opción por un derecho más limitado a beneficiarse (pasivamente) de ella. Pero el concepto de beneficios de la ciencia se recuperó por consenso, sumándose al texto chino y no sustituyéndolo" ${ }^{9}$ lo que tuvo consecuencias más importantes de lo que puede parecer.

\section{Alcance del derecho a la ciencia}

Este debate bien puede explicar la idea que a menudo tenemos de este derecho sólo como una forma de participar de los beneficios de la ciencia; por ejemplo, a tener acceso asequible a medicamentos. Pero queremos hacer una lectura más amplia y más ambiciosa que la mera idea de recibir pasivamente. El derecho a la ciencia va más allá de la visión de «beneficiarse» y aboga por contenidos más amplios de «participación»; es un derecho que incluye contenidos como la divulgación, la participación

dental: «El Dr. Chang fue uno de los pocos miembros de la Comisión que de manera constante recordó a sus colegas que una Declaración Universal debía incorporar otros sistemas filosóficos aparte de los de Occidente, y él mismo a menudo citó principios confucianos para informar la discusión. Humphrey le reconoce su dominio de la filosofía confuciana para encontrar un lenguaje de compromiso en puntos particularmente difíciles» (Johnson y Symonides, 1998: 22; traducción de los editores). La importancia de la aportación de Chang fue indicada en un sentido distinto al que aquí se sugiere por Claude: «el tono elitista del texto original, al parecer sólo para proteger a los científicos, fue así superado por la propuesta de Chang de que todos tienen el derecho a participar en los avances de la ciencia (científicos y estudiantes de ciencia) y de sus beneficios (el público en general)» (Claude 2002: 253-254; traducción de los editores).

9. Es posible que esto sea simplemente un resultado vestigial, no completamente intencional, propio de las lógicas redactoras de un texto internacional, pero no deja de ser un afortunado equilibrio que debemos aprovechar cuando, como es bien sabido, el derecho internacional no nos condena a la voluntad de los negociadores, sino que nos anima a interpretar el texto «conforme al sentido corriente que haya de atribuirse a los términos [...] y teniendo en cuenta su objeto y fin» (artículo 31 de la Convención de Viena sobre el Derecho de los Tratados de 1969). 
en la creación científica ${ }^{10}$ (citizen science) $)^{11}$, la participación en las políticas científicas $^{12}$, la responsabilidad en la gestión de riesgos ${ }^{13}$, las libertades y responsabilidades de quienes hacen ciencia y tantos otros aspectos, como veremos, adicionales al «beneficiarse de las aplicaciones» científicas.

Este mismo debate entre una participación multidimensional o un disfrute más pasivo puede aplicarse al Pacto Internacional de Derechos Económicos, Sociales y Culturales. La formulación aparentemente más restrictiva con que comienza el artículo — «Gozar de los beneficios del progreso científico y de sus aplicaciones» (artículo 15, 1 b) -, debe ser interpretada conjuntamente con el más amplio mandato que inmediatamente se añade de que «los Estados partes en el presente Pacto deberán adoptar (medidas para) la conservación, el desarrollo y la difusión de la ciencia y de la cultura» (artículo $15,2)^{14}$, el compromiso de «respetar la indispensable libertad para la investigación científica» (artículo 15, 3) para garantizar el «el respeto por la libertad indispensable para la investigación científica y la actividad creativa» y el «fomento y desarrollo de la cooperación y de las relaciones internacionales en cuestiones científicas» (artículo 15, 4).

Como se ve, son suficientes elementos como para que también en relación al artículo 15 del Pacto Internacional de Derechos Económicos, Sociales y Culturales, como hicimos en el caso del artículo 27 de la Declaración Universal de los Derechos Humanos, defendamos la idea de un derecho mucho más amplio que la idea restrictiva de beneficiarse pasivamente de los avances científicos y tecnológicos.

\section{Situación institucional actual}

Ya en el siglo XXI, una iniciativa conjunta de la Unesco, del Amsterdam Center for International Law y del Irish Centre for Human Rights, iniciada en 2007, supuso el inicio

10. «Se va abriendo paso la idea de que el saber es un asunto de todos, una tarea a la que [...] contribuyen todos los ciudadanos. Poco a poco ha ido reconociéndose a todos los seres humanos la capacidad de participar en la investigación, inventar y hacer ciencia, o al menos juzgar algunas de sus conclusiones. A principios de los noventa, organizaciones como la American Association for the Advancement of Science (AAAS) y la Unesco propagaban el lema «Science for all», resumido en el siguiente principio: no sólo ciencia al servicio de todos, sino ciencia por todos» (Innerarity, 2011: 130).

11. «La idea de una 'ciencia cívica' [...] o de una 'ciudadanía científica' [...] alude a los actuales desafíos acerca de cómo introducir a los agentes no científicos en los procesos de decisión, cómo tener en consideración el saber y la experiencia local, cómo realizar una comunicación transparente del riesgo u otras exigencias de democratización similares» (Innerarity, 2011: 114).

12. Sobre la participación en las decisiones y sobre sus obstáculos y formas de superarlos, con importantes ideas e interesantes ejemplos, véase Kleinman (2000).

13. Sobre el principio de precaución, que se presta muy fácilmente a debates maniqueos, véase el inteligente planteamiento de Whiteside (2006) en el capítulo «Science or Precaution? A Misleading Dichotomy»: frente a una visión simplista despliega una «panoplia de medidas de precaución».

14. Sobre el significado de estos importantes contenidos adicionales, véase Claude (2002: 259-260). 
del regreso del derecho a la ciencia al escenario internacional. Hasta esa fecha sólo algunos autores aislados y algunas pocas organizaciones científicas habían mostrado interés ${ }^{15}$.

La Unesco y los centros citados organizaron en el 2007 una reunión de expertos en Ámsterdam en cuyas conclusiones se tocaban bastantes de los puntos tratados en este artículo. Además se propusieron importantes acciones de seguimiento que en parte se han cumplido y en parte quedan aún por cumplir, pero que siguen teniendo sentido. Estos actores llamaron a «una coalición para la promoción del derecho [a la ciencia]» cuyo gran objetivo sería el debate sobre el contenido normativo del derecho, incluyendo a todos los agentes relevantes ${ }^{16}$.

A este encuentro siguieron otros dos de las mismas características en Galway (2008), Irlanda, y Venecia (2009). Hoy los trabajos presentados en estos tres encuentros, así como sus conclusiones y declaraciones finales, forman un corpus de imprescindible consulta para quien quiera tratar de este asunto.

Farida Shaheed, experta independiente sobre derechos culturales, tomó el testigo y presentó ante el Consejo de Derechos Humanos, en su vigésima sesión, un informe temático sobre este asunto (Asamblea General de las Naciones Unidas, 2012a). Este informe pudo completarse tras un proceso de consulta en el que participaron 21 Estados y 13 organizaciones.

Este informe supuso un importante paso adelante en el proceso iniciado por la Unesco y sus socios para el desarrollo del derecho humano a la ciencia y la definición de sus contenidos. La relatora recomendó al Comité de Derechos Económicos, Sociales y Culturales «revisar el artículo 15 de una manera integral y considerar la adopción de un nuevo Comentario General que recogiera todos los derechos recogidos en ese artículo» (Asamblea General de las Naciones Unidas, 2012a: 21).

En octubre de 2013, la Oficina de la Alta Comisionada organizó, por mandato del Consejo de Derecho Humanos (Asamblea General de Naciones Unidas, 2012b) un seminario de dos días sobre el artículo 15.1 b), en Ginebra, con el objetivo de «facilitar clarificación adicional sobre el contenido normativo del derecho a gozar del desarrollo científico y sus aplicaciones; y clarificar su relación con otros derechos humanos». En este seminario se unieron varias voces al llamado al Comité de Derechos Económicos, Sociales y Culturales para avanzar hacia un Comentario General sobre el derecho a participar en los beneficios que resulten del avance científico y de sus aplicaciones.

15. La Unesco había trabajado sobre la cuestión de una forma más bien indirecta o con enfoques temáticos muy especializados (bioética y genoma humano). Para un estudio de la labor de la Unesco en la implementación de este derecho entre 1948 y 1998, véase Symonides (1998: 83-84).

16. Véase «Report of the Unesco Experts' Meeting on the Right to Enjoy the Benefits of Scientific Progress and its Application», (Amsterdam, 2007), disponible en http://bit.ly/2vYAPSO. 
El Comité de Derechos Económicos, Sociales y Culturales asumió su tarea y se encuentra en este momento debatiendo los borradores de un futuro Comentario General sobre el Derecho a la Ciencia que deberá determinar el contenido normativo de este derecho y orientar a los Estados, en particular, y a la sociedad, en general.

\section{Contenido normativo}

Como queda dicho, el contenido normativo de este derecho será el objeto del Comentario General, pero aquí podemos presentar algunas de las ideas ya aportadas por los eminentes expertos que han tratado hasta la fecha el asunto.

Aquí me basaré en las ideas, aportaciones y propuestas presentadas por Claude (2002), Schabas (2007), Chapman (2009), Donders (2009), Shaver (2010), Shaheed (Asamblea General de las Naciones Unidas, 2012a) y Margaret Weigers Vitullo y Jessica Wyndham (AAAS Science and Human Rights Coalition, 2013).

Claude (2002) realizó un importantísimo primer acercamiento a la cuestión que nos ocupa, en el que ya tocaba muchos de los puntos que vamos a ver en otros autores y con la ventaja añadida de que incluía importantes ejemplos y casos prácticos para cada tipo de obligación o tipo de vulneración. El primer esfuerzo de Claude incluía, además, un muy rico planteamiento que ponía en relación el artículo 15.1 b) del derecho a participar en los beneficios que resulten del avance científico y de sus aplicaciones con el resto de contenido del artículo 15. El trabajo de Claude sigue siendo actual.

En 2009 se celebró la reunión de expertos de Venecia donde Donders (2009: 9) analizó el derecho mediante una interpretación textual y aplicando el marco conceptual que había sido ya empleado por el Comité de Derechos Económicos, Sociales y Culturales para otros derechos. Donders propuso cuatro contenidos del derecho, a saber: 1) libertad científica; 2) el derecho a ser protegido de posibles efectos dañinos de la ciencia; 3 ) el acceso (en el que incluye ciertos elementos de participación); y 4) la cooperación internacional.

Tanto Donders (2009), como Chapman (2009), Shaver (2010) y la AAAS Science and Human Rights Coalition (2013) adoptan a la interpretación de este derecho dos de los criterios que ya habían sido adoptados por el Comité de Derechos Económicos, Sociales y Culturales en las observaciones generales relativas a otros importantes derechos: el esquema de triple tipología de obligaciones ${ }^{17} \mathrm{y}$, con distintos matices, los criterios de disponibilidad, accesibilidad, aceptabilidad, calidad y adaptabilidad.

Chapman (2009: 18-57) desarrolla en extenso la aplicación de la ya clásica triple tipología de obligaciones (respetar, proteger y hacer efectivos) al caso del derecho a la ciencia. Igualmente interesantes son las consideraciones que la autora hace sobre

17. En la Declaración de Venecia se incorporan cuatro puntos (del 13 al 17) sobre el contenido normativo que recogen también el esquema de triple obligación. 
la relación de este derecho con las cuestiones de participación, transparencia y rendición de cuentas (2009: 15-16).

Shaver (2010:167) enumera los conceptos necesarios para la interpretación de este derecho, que deben incluir, entre otros, la «no discriminación, realización progresiva y obligaciones básicas esenciales».

Farida Shaheed presentó, como ha quedado dicho, en 2012 su informe ante el Consejo de Derechos Humanos, en el que dedica veinticuatro numerales al contenido normativo del derecho, y que recogen mucho de lo indicado por los autores citados. Estos numerales suponen todo un corpus básico que será fundamento indispensable para los siguientes intentos «onusianos» de desarrollar este derecho. A los efectos de este trabajo, valga decir aquí que, para la experta independiente, este contenido normativo debe contener: a) el acceso a los beneficio de la ciencia por parte de todos, sin discriminación; b) oportunidades para todos de participar en la empresa científica y la libertad indispensable para la investigación científica; c) la participación de las personas y las comunidades en los proceso de toma de decisiones; y d) facilitar un entorno que favorezca la conservación, el desarrollo y la difusión de la ciencia y la tecnología (AAAS Science and Human Rights Coalition, 2013: 9).

\section{Conclusión}

En conclusión, estamos ante un derecho que puede denominarse ya «derecho a la ciencia» y que tiene contenidos de derecho de participación, derecho de acceso (que implica libertades) y derecho con importantes claves de cooperación internacional. Confío en que en unos meses podamos ver ya aprobado el Comentario General del Comité de Derechos Económicos, Sociales y Culturales, del que tengo el honor de ser relator, y ver en él recogidas algunas de las ideas que en este artículo hemos comentado.

Este futuro comentario general debe servir de guía a los Estados, pero también debe aportar elementos a las organizaciones científicas, los científicos y la sociedad civil, en general, para conocer mejor sus derechos y sus responsabilidades.

\section{Referencias}

AAAS Science and Human Rights Coalition (2013). «Defining the right to enjoy the benefits of scientific progress and its applications: American scientists' perspectives». Informe preparado por Margaret Weigers Vitullo y Jessica Wyndham. Disponible en http://bit.ly/2eFMSkO.

Asamblea General de las Naciones Unidas (2012a). Report of the Special Rapporteur in the field of cultural rights, Farida Shaheed. The right to enjoy the benefits of scientific progress and its applications. Documento A/HRC/20/26. Disponible en http://bit.ly/2tRBqnS. 
-. (2012b). Promoción del disfrute de los derechos culturales de todos y respeto de la diversidad cultural de 2 de julio de 2012 (A/HRC/20/L.18). Disponible en http:// bit.ly/2tIokuV.

Chapman, Audrey R. (2009). «Towards an understanding of the right to enjoy the benefits of scientific progress and its applications». Journal of Human Rights, 8 (1): 1-36.

Claude, Richard Pierre (2002). «Scientists rights and the human right to the benefits of science». En Audrey Chapman y Sage Russel (editors), Core obligations: Building a framework for economic, social and cultural rights (pp. 247-278). Amberes, Oxford: Intersentia.

Donders, Yvonne (2009). «Right to enjoy the benefits of the scientific and technological progress and its aplications». Report of the Experts' Meeting. París: Unesco.

Glendon, Mary Ann. (2001). A World Made New: Eleanor Roosevelt and the Universal Declaration of Human Rights. Nueva Iork: Random House.

InNerarity, Daniel (2011). La democracia del conocimiento. Por una sociedad inteligente. Barcelona: Paidós.

Johnson, M. Glen y Janusz Symonides (eds.) (1998). The Universal Declaration of Human Rights A history of its creation and implementation 1948-1998. París: Unesco.

Kleinman, Daniel Lee (2000). «Democratization of science and technology». En Daniel Lee Kleinman (editor), Science, technology and democracy (pp. 139-165). Nueva York: State University of New York Press.

Mancisidor, Mikel (2010). «Los derechos económicos, sociales y culturales: Una puesta al día». En Manuela Mesa Peinado (coordinadora), Anuario CEIPAZ 20102011. Balance de una década de paz y conflictos: Tensiones y retos en el sistema internacional (pp. 105-127). Barcelona: Icaria.

-. (2015a). «Is there such a thing as a human right to science in international law?». ESIL Reflections, 4 (1): 1-6. Disponible en http://www.esil-sedi.eu/node/896.

-. (2015b). «Historia del derecho humano a la ciencia». En Niko Huhle y Teresa Huhle (editores), Die Subversive Kraft der Menchenrechte. Rainer Huhle zum radikalen Jubiläum. Oldenburg: Paulo Freire Verlag.

MoRsink, Johannes (19999). The Universal Declaration of Human Rights: Origins, drafting and intent. Filadelfia: University of Pennsylvania Press.

Preston, Diana (2005). Before the fall-out. From Marie Curie to Hiroshima. Nueva York: Walker \& Company.

Shaver, Lea (2010). "The right to science and culture». Wisconsin Law Review, 1: 121-184.

Schabas, William A. (2007). «Study of the right to enjoy the benefits of the scientific and technological progress and its aplications». En Yvonne Donders y Vladimir Volodin (editores), Human Rights in education, science and culture (pp. 273-308). París: Unesco. 
-. (2013). The Universal Declaration of Human Rights. The travaux préparatoires. Cambridge: Cambridge University Press.

Symonides, Janusz (1998). «Unesco and the Universal Declaration of Human Rights».

En M. Glen Johnson y Janusz Symonides (editores), The Universal Declaration of Human Rights A history of its creation and implementation 1948-1998 (pp. 77-110). París: Unesco.

Whiteside, Kerry H. (2006) Precautionary politics. Principle and practice in confronting environmental risk. Cambridge, Massachusetts: MIT Press.

\section{Agradecimientos}

El autor agradece a la Diputación Foral de Bizkaia el apoyo al proyecto de reconocimiento internacional del derecho humano a la ciencia. Este artículo forma parte de las actividades de investigación, divulgación e incidencia de dicho proyecto.

\section{Sobre el autor}

Mikel Mancisidor es licenciado en Derecho por la Universidad de Deusto y doctor en Relaciones Internacionales por la Geneva School of Diplomacy and International Relations. Es profesor adjunto del Washington College of Law de la American University y miembro del Comité de Derechos Económicos. Relator para la Observación General sobre el «Derecho Humano a la Ciencia». Su correo electrónico es mikel. mancisidor@deusto.es. 
